\title{
Visible Light Communication Systems Conception and VIDAS
}

\author{
Navin Kumar, Nuno Lourenço², Michal Spiez ${ }^{2}$ and Rui L. Aguiar ${ }^{1,2}$ \\ ${ }^{1}$ Telecommunications Institute, Campus Universitário de Santiago, 3800 Aveiro, Portugal. \\ ${ }^{2}$ Electronics Telecommunications and Informatics Department, University of Aveiro, \\ Campus Universitário de Santiago, 3800 Aveiro, Portugal.
}

\begin{abstract}
Visible Light Communication (VLC) using LEDs is emerging as a key technology for a ubiquitous communication system, because LED has the advantages of fast switching, long life expectancy, being less expensive and being visible light that is safe for the human body. The VLC system is expected to undergo rapid progress, inspiring numerous indoor and outdoor applications; however, many technical issues need to be addressed, especially in outdoor environment. In order to provide a better understanding of the research challenges of VLC, this article presents a detailed investigation of the current state-of-art concept. Open research issues such as channel modelling and modulation techniques are also discussed, with the objective of triggering new research interest in this field. The paper also introduces our ongoing development of Wireless USB Interface and the project VIDAS.
\end{abstract}

\section{Keywords}

VIDAS, Visible light communication, Wireless optical communication.

\section{Introduction}

Colored and white LED (light emitting diode) devices are being used in large scale in indoor lighting, full color displays, traffic lights, car lights etc. Compared with conventional lighting methods, high brightness LEDs have higher power efficiency, long life expectancies, higher tolerance to humidity, lower heat generation and smaller sizes, which make these devices strong candidates for present and future lighting technology [1,2]. Fortunately, an intrinsic characteristic of LED is that it is a semiconductor, with high rate switching capabilities, making it possible to extend its usage to data transfer in wireless communications systems, usually referred as Visible Light Communications.

The VLC concept directly sprouts from the use of optical spectrum in indoor wireless communication systems, initially introduced in 1978 by Gfeller [3] through the utilization of infrared wavelengths in nondirected wireless communications. Since then, many developments have cropped up, covering both point-to-point systems - handled in organizations such as IrDA (Infrared Data Association) - and in nondirected local area networks such as the early IEEE802.11 standards. Despite its constraints in infrared wireless communications, infrared is recognized as offering a major advantage over radio technologies for wireless systems: a large, virtually unlimited, bandwidth that is unregulated worldwide [4-7]. With recent high power colored and white LEDs, these advantages can be also explored by VLC systems. Akanegawa [8] discussed the use of LEDs for traffic lights; Pang [9] proposed an optical wireless broadcasting system based on control traffic lights using colored high brightness LEDs and Komine [10-12] presented the integration of white LED VLC into Power-Line Communications (PLC). These authors were pioneers proposing the utilization of white and colored LEDs to transmit data when, previously, these devices were being used only for lighting and signaling applications.

The present paper addresses the utilization of VLC in multi-Megabit communications, including a wireless extender of a USB interface and a wireless Advanced Driver Assistance System (ADAS), to increase traffic safety. These systems present particular properties, since they are focused on different scenarios. While the wireless USB interfaces extender is usually confined to indoor environments, the wireless ADAS is an outdoor application. There has been very little work on the use of visible light as a communication medium. High brightness LED devices, when used in indoor illumination or in outdoor applications, present many unsolved challenges.

In free space environment, the luminous intensity and transmitted optical power are the two basic characteristics of LED lights. The luminous intensity is used for expressing the brightness of an LED, while the 
transmitted optical power indicates the total energy radiated from an LED. The LED brightness and optical power will be one of the deciding criteria for the range of data communication in free space. Though in the indoor environment, line-of-sight (LOS) as well as diffused links can be used for VLC, in the outdoor, it relies mostly on LOS link. Therefore, range of communication is definitely a limitation of VLC, especially in the outdoor environment. The optical signal from LED transmitter is then intensity modulated (IM) with Direct Detection (DD) and generally On-Off Keying (OOK) modulation scheme is used [13] to send information.

This paper presents a brief introduction to VLC systems. Section 2 presents a conceptual VLC system architecture. The next section deals with the state-of-the art of VLC and its applications. A wireless USB interface based on VLC is presented in section 4 while section 5 presents the future challenges and an introduction to a national project titled VIDAS. Finally, section 6 reports the concluding remarks of this work.

\section{Visible Light Communication Systems}

\subsection{General System Architecture}

In this section, we introduce a basic VLC system using LEDs for indoor application, as shown in Figure 1. In this system, there are two kinds of lighting devices. The one used in optical downlink is composed of many plural white LEDs, replacing incandescent lamps on the ceiling and generally has a large superficial area and a wide angle of irradiance. Another lighting

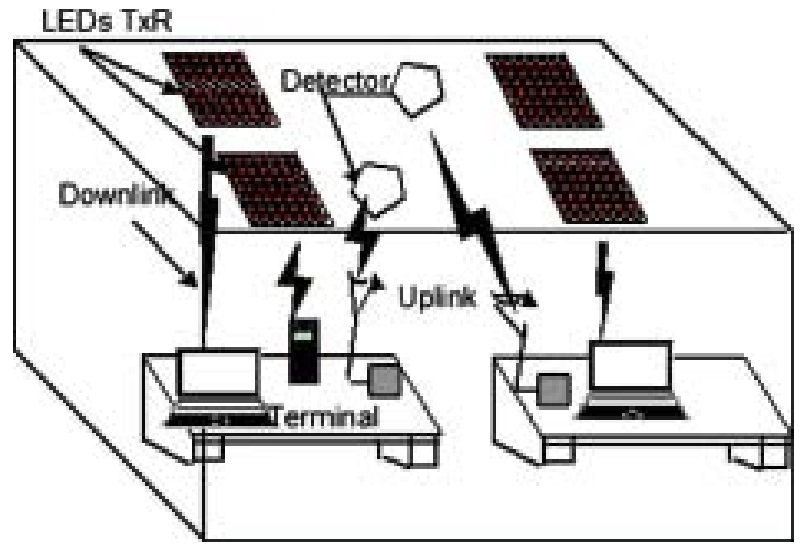

Figure 1: Simple general VLC for indoor application.

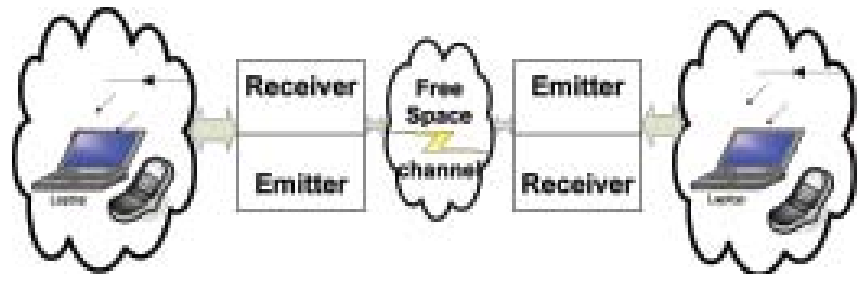

Figure 2: Overall block diagram of a VLC. device, used in optical uplink, is composed of several white LEDs. It has a small superficial area and a narrow angle of irradiance, like an electric torch. From Figure 1, we can see data transmission from the lighting device LEDs to a PC equipped with a detector in the downlink and from a table lamp to a detector in the uplink.

This dual function is based on the fast switching of LEDs and the modulation of the visible light waves for wireless communications. Figure 2 shows the block diagram of the system model applicable for indoor environment.

This system consists of a light source which emits light and data simultaneously. Data is sent between two or more terminals; in each terminal there is a receiver and an emitter. The emitter transmits data into free space, to be received by a receiver from a different terminal. Block diagram of the transmitter is given in Figure 3.

At first, we modulate information into the luminance and then transmit the information by blinking LED. For optical wireless links, the most viable modulation is intensity modulation (IM), in which the desired waveform is modulated onto the instantaneous power of the carrier.

Next, we receive the information by capturing the blinking transmitter. The most practical down-conversion technique is direct detection (DD). A photodiode is generally applied as a light sensor. The receiver block is as shown in Figure 4.

The data rate in VLC is limited by the switching speed of LEDs transmitter. In the indoor environment, the

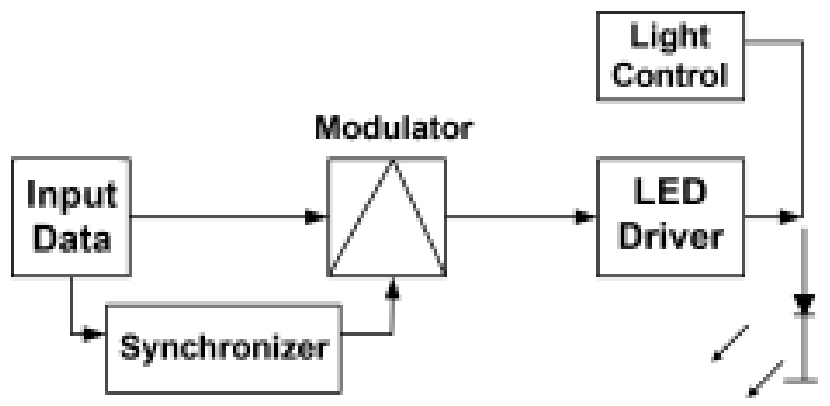

Figure 3: Transmitter blocks.

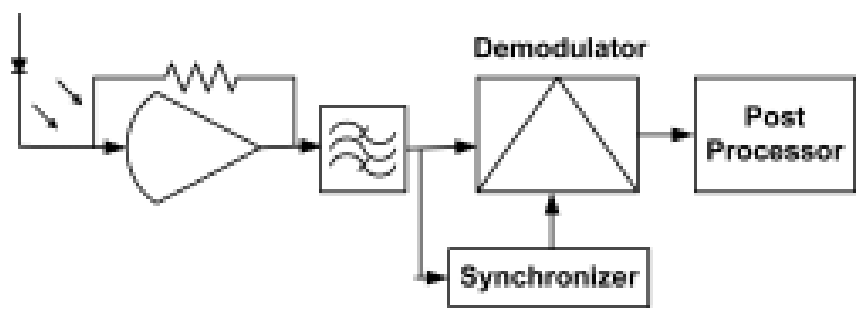

Figure 4: Receiver block. 
experiment has been performed at $1 \mathrm{Mbps}$ with OOK. In the outdoor, for short range within 20-30 meters at $1 \mathrm{Mbps}$, to maintain Bit Error Rate (BER) of $10^{-6}$, it is expected that an SNR of around $14 \mathrm{~dB}$ should be maintained. However, in our application, we have a target of around 70-90 meters of range, where data rate is not an important issue. In the intended application, a data rate of $128-256 \mathrm{Kbps}$ would be sufficient within the limitation of around A4 size page information per second time period, suitable to be detected and interpreted by recipients.

The VLC system, in fact, needs a complex channel characterization, modulation and coding schemes. This working group at this institute is devoted to investigate and is expected to design the best system for a particular environment under the project VIDAS, introduced in section 4 .

\section{VLC State of the Art and Applications}

This section presents a short overview on the recent trends towards VLC design and applications. Section 3.1 describes the main research efforts and results. Section 3.2 describes some VLC applications, reflecting their usage for both indoor and outdoor environments. Section 3.3 presents an overview on the main research opportunities, disclosed by the ever growing high brightness LED industry. Finally, section 4.1 discusses the impact of modulation schemes on the overall VLC system performance.

\subsection{Research Efforts}

The Visible Light Communication is a novel kind of optical wireless communication, utilizing white LEDs. The use of visible light as a communication medium is still at a very early stage, compared to what has been achieved in areas like infrared and laser (free space optics). The VLC system using LEDs as a light source has many distinctive features and has high potential to be a ubiquitous communication system. Despite this, it has not been investigated deeply. In [14], the authors propose an indoor visible light data transmission system, as shown in Figure 5, which utilizes white LEDs light. There has been considerable progress on research as far as indoor visible light communication is concerned. An audio system and novel use of LEDs for audio and data transmission was proposed in [8]. It is interesting to notice that both the LED industry and VLC research interest have undergone an exceptional increase in Japan. The main reason for this is the fact that major LED industries are presently placed in Japan. More than twenty companies participated in the VLCC (Visible Light Communication Consortium) held in Japan, in 2003 [15]. In 2006, they created a standard 'Visible Light Tag' for low data rate application such as sending various ID from LED lights.

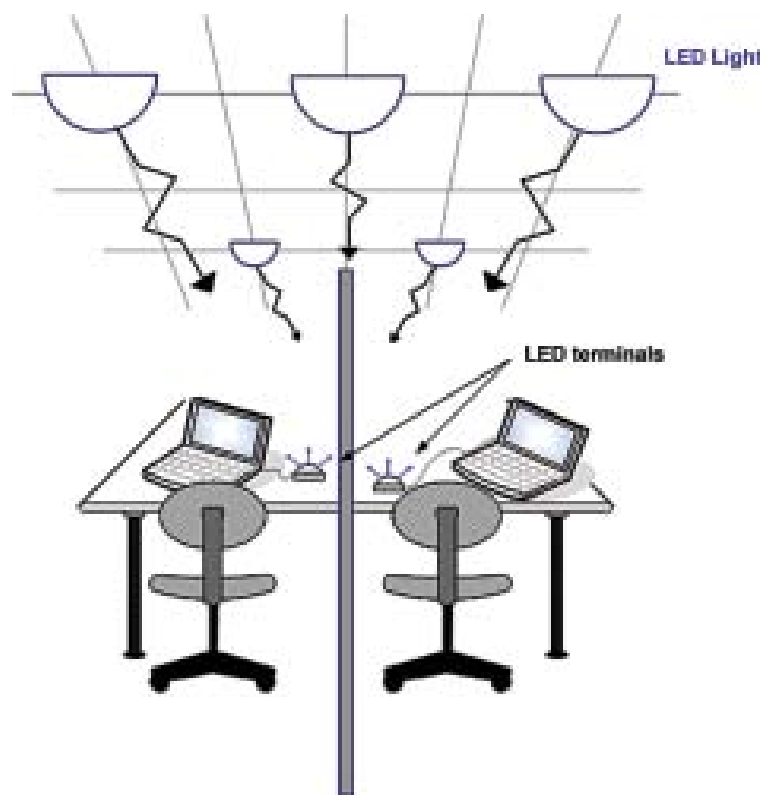

Figure 5: An indoor light arrangement.

In outdoor situations, the scenario is different. Akanegawa and Maehara, in [7], conducted theoretical tests on visible light communication using an LED traffic signal. Hayashi [16] undertook field experiments on visible light communication, using a pedestrian LED traffic signal for a distance of about $30 \mathrm{~m}$, without considering the communication speed. Another interesting area for research and application is Parallel Wireless Optical Communication (PWOC) $[17,18]$. In this, the authors have suggested the use of high-speed camera as a receiver, which can use visible light communication for road-to-vehicle communication, which will assist drivers by providing various kinds of information. However, to sum up, there has been very little work on the use of visible light as a communication medium.

\subsection{Applications}

The visible light communication is more advantageous than radio and infrared communications in terms of ubiquitousness, transmission at ultra high speed and harmlessness for the human body and electronic devices. We describe here a few applications of this technology, that have opened up new dimensions of research. This technology can act as a standard light, but stand underneath it with a suitably-equipped gadget, such as a PDA (personal digital assistant), mobile phone or MP3 player, and data can be instantly transferred straight to the gadget, at speeds approaching that of optical fibre. Taking the advantage of using the existing infrastructure around us, the transmission of positional information entered into individual indoor LED lighting devices and sent to a cellular telephone or similar devices enables position detection that is accurate, to within several meters. Visible light communications allow one to easily 
receive on-off positioning information, such as 'where one is' and so on, from illumination and emergency lights positioned here and there. Simply holding a handset up to a large screen will facilitate transmit a telephone's ID. Also, merely pointing the phone in the direction of the desired information on-screen could cause relevant information to be downloaded onto the phone. There can be many more applications: at exhibitions, where we can stand underneath a VLC lamp and download information from the display; in cameras and camera phones, with the optics from the camera lens being used to receive the data; in the office, with a VLC-equipped desk-lamp being used as a modem for our laptop, almost any place where we currently use WiFi.

It would be ideal for sports broadcasts or live TV interviews. Much of the free-space optics has been concentrating on data transfer point-to-point, but we can spread it across the whole city. Musicians each could play a different instrument under red, green and blue lights. When visitors wearing headphones turn in the direction of one of these lights, they will be able to hear only the sound of the instrument receiving that particular coloured light. By making the most of the visibility of this light, visitors will be able to instinctively choose the information that they want to receive.

Additionally, this system could have a leg up on other wireless alternatives in sensitive areas such as hospitals, as the VLC creation will not interfere with pulse-makers and other medical equipment; it could be used as a visible light tag under water. We may call such visible light communications as ubiquitous light communications, integration between visible light communications and ubiquities computing. There are numerous applications of VLC in both indoor and outdoor environments.

\subsection{Light Emitting Devices}

Significant developments in aluminium gallium indium phosphide (AlGaInP) technology have seen red and amber LEDs penetrate the automotive and traffic signal markets. Recently, novel high-power AlGaInP LEDs employing means for high extraction efficiency have been developed, culminating in a world record performance for red-orange LEDs $(>1001 \mathrm{~m} / \mathrm{W})$ that, for the first time, exceeded the luminous efficiency of most fluorescent bulbs. These LEDs have been coupled with down-converting luminescent phosphors to provide white light LEDs, with efficiencies $(30 \mathrm{~lm} / \mathrm{W})$ now exceeding that of incandescent and halogen bulbs. With best-reported wall-plug efficiencies of $45 \%$ (AlGaInP) and $25 \%$ aluminium gallium indium nitride ( $\mathrm{AlGaInN}$ ), we can look forward to further significant improvements in LED efficiency and light output in the coming years, opening more doors towards a new era of solid-state lighting.

The LED has been studied as a future energy-saving light source. In the experimental demonstration, authors in [19] tested the life of LEDs from the same manufacturer, as compared to that from a different one and they also suggested a design perspective on how to increase the life for LEDs. Multichip LED lamps [19] offer many advantages such as chromaticity control, better light quality, and higher efficiency. Recent advancement in power flip chips, including lamp reliability, white LED technology, and what the future might hold for Illumination with Solid State Lighting Technology have been summarized in [21].

The most important parameters for the design of LEDs for optical communication are frequency response and efficiency. Table I in Appendix A shows the frequency and switching response as a result of tests, carried out at our lab, on LEDs from two different manufacturers.

Concurrent use of visible LEDs for simultaneous signalling and communication, called iLight, leads to many new and interesting applications and is based on the idea of fast switching of LEDs and the modulation of the visiblelight waves for free-space communications.

\section{Wireless USB Interface}

This section describes an optical USB interface. This interface is used as a demonstrator of the VLC indoor potentiality. It would allow users to connect any USB plug in device remotely, using a VLC system. An overall system architecture is shown in Figure 6.

The VLC Wireless USB interface is designed to allow to different terminals (PC, notebook, cell phone, PDA etc) to connect themselves using visible light as the medium of communication. Using the popular USB interface, available in almost every fixed or mobile terminal, people can attach a wireless communication module without
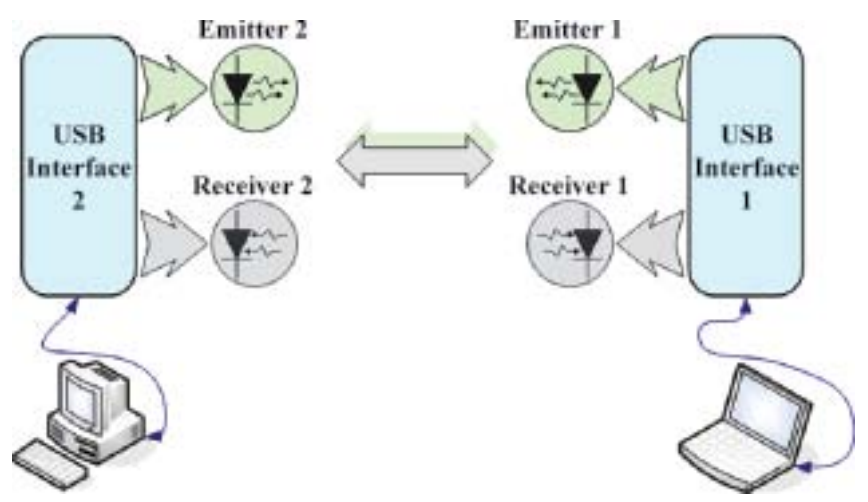

Figure 6: System architecture. 
needing to make complicated configurations or connections. A single device contains a LED transmitter, a photodiode receiver and an USB interface adapter, based on a FPGA. Trying to make a direct coupling of the emitter and the receiver to the terminal would require complex electronics; so using an FPGA based solution allows us to build an adaptable solution that can be easily upgraded.

The receiver and emitter are controlled by the FPGA interface adapter through a group of status indicators and control signals. This control is necessary to ensure that the data transmission/reception is done in the best of conditions. For example, if the wireless module is receiving, it should not be able to transmit; otherwise, the noise generated by the emitter LED could corrupt the received data. In the USB demonstrator module, we can use a simple enable/disable line to switch $\mathrm{ON}$ and OFF the receiver or the emitter, but we need to control the remaining devices within the network. For a simple demonstration network, High-Level Data Link Control (HDLC) is one of the best data-link layer protocols. It is bit-oriented and provides connection-oriented or connectionless service. High-Level Data Link Control (HDLC) links can be classified as unbalanced or balanced; in the first case, we have one primary terminal and one or several secondary terminals, while in the second case, we have two peer terminals. The link configuration more suited for our demonstration network is Asynchronous Response Mode (ARM), which is an unbalanced configuration in which we can have the secondary terminals transmitting without the permission of the primary terminal. Nevertheless, the primary terminal is still responsible for initializing the transmission line, recovering from errors and logically disconnect the devices. Such a configuration would allow us to integrate several USB modules in an office or home network, without having excess data loss due to noise interference by transmitting data to an eventually receiver in OFF. However, in the case where more nodes are contending for the channel or trying to send data, collision may occur, resulting in packet drop and loss. The contention mechanism such as RTS/CTS (Request to Send/Clear to Send) can then be used. In this case, a node wishing to send data initiates the process by sending a Request to Send frame. The destination node replies with a Clear to Send frame. Any other node receiving the RTS or CTS frame should refrain from sending data for a given time. The amount of time the node should wait before trying to get access to the medium is included in both the RTS and the CTS frame. The RTS/CTS mechanism reduces frame collisions during system operation.

\subsection{Emitter}

By considering a simple emitter for an USB interface application, we can have two main options: (i) a simple LED topology, or (ii) an LED array topology. We present

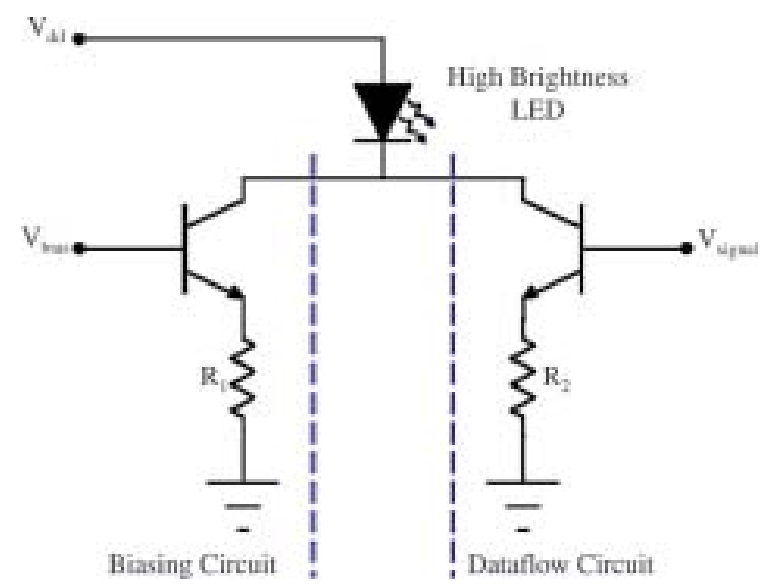

Figure 7: Emitter topology.

the simple LED topology, because it is the basic concept for the emitter design. As can be seen in Figure 7, the device has two main circuits responsible for the LED driving current.

The biasing circuit is responsible for driving a constant current through the LED. This is important in integration of the data transmission circuit with an illumination system. The data flow circuit allows us to control the LED current and, in turn, its brightness, so that we can transmit information through the variation of the LED brightness level, thereby increasing the system performance. To increase the backlight level, we would need to adjust the value of $\mathrm{R} 1$ in Figure 7 above or to increase the strength of the data signal, we would need to increase the current controlled by the value of R2 in Figure 7 above. Although we have used bipolar transistors in the design shown, we could have also used MOS transistors. Nevertheless, the necessary changes to be made to, for example, an off the shelf LED array lamp, are very simple, making this an interesting topology to use. There are several variations, especially regarding the filtering of the AC current source that can make the emitter more or less efficient. The combined heat from the high brightness LED and the current control transistors can reach significant values; therefore, the use of an external dissipater may be necessary.

\subsection{Receiver}

One of the possible designs for the VLC receiver is shown in Figure 8 below. The proposed design uses a positiveintrinsic-negative (PIN) photodiode. It does not have a high gain such has the avalanche photodiode (APD), but it can become an advantage in high noise environments, where the APD tends to saturate. The PIN is also cheaper and has a larger active area.

In order to receive the maximum amount of signal we 
Kumar N, et al.: Visible Light Communication Systems

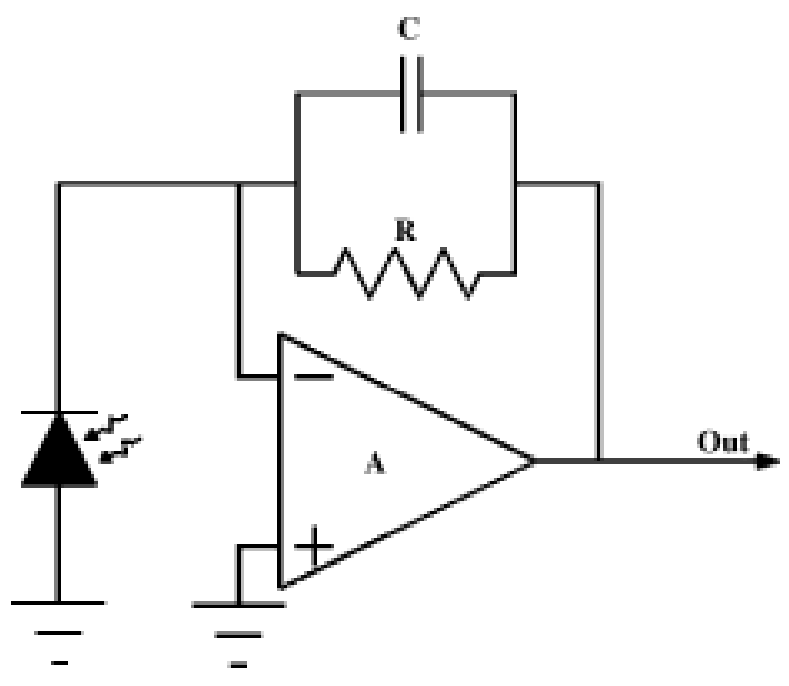

Figure 8: Receiver topology.

need a large active area, but devices with large active areas also have a high internal capacitance. We could also use an array of small PIN photodiodes connected in parallel; however, the value of the final equivalent capacitance has to be kept in mind.

The noise interference due to other light sources, all with different spectral power densities, is another challenge in this design. Incandescent lights emit high levels of infrared radiation; fluorescent lamps emit higher levels in the visible range, while the sun emits not only high levels of ultraviolet radiation but also a considerable amount of infrared radiation. One possible solution for evading these noise sources would be the use of an interference filter. This filter, placed on the PIN photodiode, would allow the receiver to receive only signals in a predetermined wavelength band. Nevertheless, the use of such a filter would reduce the strength of the received signal, making the device more susceptible to receiving errors.

\subsection{USB Interface}

Wide uses and applications of Universal Serial Bus (USB) motivated developers and manufacturers from big companies to form a Promoter Group in 2004, to define Wireless USB (WUSB) standard. The basic technology that can support the desired applications and compete with the USB is Multiband Orthogonal Frequency Division Multiplexing (Multiband OFDM), which is again limited by bandwidth and is subject to spectrum regulation. Their development has not taken off as expected and it is delayed because of various implications such as backward compatibility, rate of transfer to at least USB $2.0(480364 \mathrm{Mbps})$ and so on. Meanwhile, this group at IT Aveiro could visualize the future of Wireless Optical Interface in Visible Spectrum and it has already started

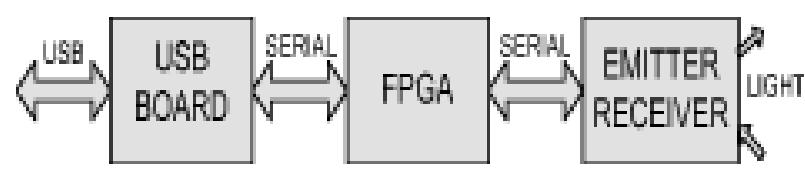

Figure 9: USB interface.

developing and testing. A situation can be visualized wherein all the devices in a home office such as the scanner, printer, external hard drive, digital camera could be connected to a PC in a way as easy as lighting a torch in search of these devices and transferring a file, video, image or printing photo instantly. It can be imagined to have an interface based on VLC that is as handy as WUSB, just a click away and that can transfer at a rate far more than an anticipated WUSB. It is like a Wireless IR incorporating many additional features. For such a system conception, where two asynchronous systems appear, digital interface is required. Buffer that levels the difference in data transfer between VLC and USB standard can be implemented within the FPGA. Digital programmable logic ensures flow of data reliably and its serial communication to USB board and transceiver guarantees fast data transmission rates. Figure 9 illustrates the design idea and block diagram of such interface.

\section{The Future Challenges in VLC and 'VIDAS'}

Optical Wireless Communication using both visible light and infrared Communication can become a viable option for last mile access and ubiquitous availability. However, where the atmospheric attenuation of optical signals and unpredictability of weather conditions limit the distance and affect link availability, high background ambient adds another big challenge that reduces signal-to-noise ratio in VLC. In the atmosphere, absorption is caused by the presence of water particles and carbon dioxide. Scattering in the presence of fog, haze, rain and snow deflects away the light and shimmer by light refraction, air density cloud, wind and atmospheric turbulence. It is possible to reduce the effect of scattering by capturing some of scattered light, using an array of detectors Field Of View (FOV). However, it would be difficult to implement it in ADAS and traffic situation.

The ADAS systems using VLC mostly rely on LOS. In traffic safety application, the failure of LOS is very common on the road, either from traffic light/road light or from vehicle to vehicle. In such cases, no traffic information can be received by the intended receiver mounted on the vehicle. Another problem is the range of communication; the LOS links limit the range. However, in some cases auto-beam tracking system may be used to increase the optical power of the transceiver to increase the footprint of the coverage. Scintillation 
is the temporal and spatial variation in light intensity caused by atmospheric turbulence such as wind and temperature gradients that create pockets of air with rapidly varying densities and, therefore, fast-changing indices of optical reflection. Therefore, VLC using LED traffic signals and an in-vehicle receiver features pose several challenging problems such as: i) the necessity of long-distance transmission; ii) the influence of a lot of optical noise sources (i.e. background lights, sun, etc..); iii) variation in atmospheric condition (such as humidity, rain drop, fog etc); iv) changes in the positional relationship between the transmitter and the receiver and many more.

VIDAS (Visible light communication for advanced Driver Assistance System) project is concerned with the investigation of the usage of outdoor illumination to increase traffic safety (which is a key issue). VIDAS is an advanced application of VLC for ADAS. Currently, several ADAS Integrated Projects, supported by the European Union (EU), such as AIDE-IP, PREVENT-IP, and SAFESPOT-IP, are addressing different ways to improve traffic safety. VIDAS is based on existing infrastructure and/or with slight changes/modifications and which, therefore, promises to be cost effective systems. Integrating VLC in Intelligent Transport System (ITS) is challenging. In ITS, LED traffic lights and LED traffic signs broadcast driving assistance data to cars (roadto-vehicle communications); LED car brake lights can transmit warning data to a car behind (vehicle-to-vehicle communications). Transmitting different information for every lane of a road is possible, as shown in Figure 10. Using the concept of Parallel Wireless Optical Communication, each of the LEDs in traffic lights, traffic signs or car brakes can be individually modulated and, thus,parallel data transmissions are possible [16] The opposing lane vehicle information system provides information on incoming vehicles to car drivers via a road information board, to draw their attention when

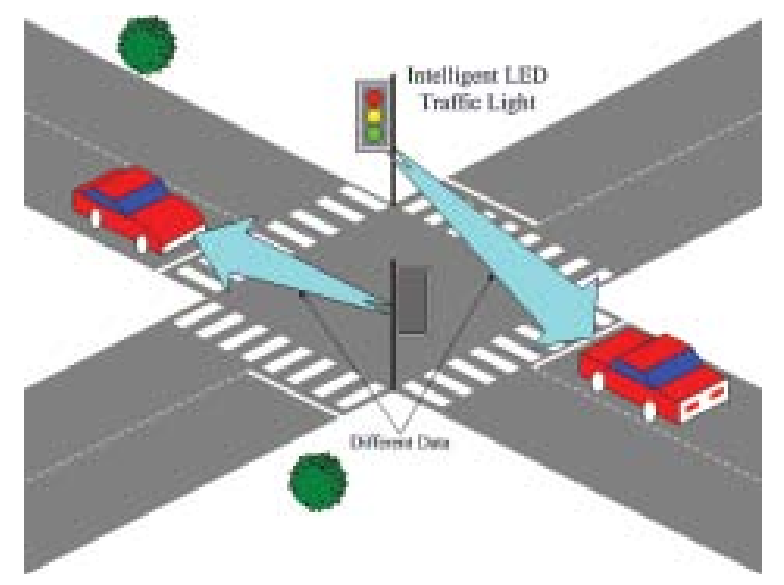

Figure 10: Visible light communication in ITS. they are approaching a curved section, after detecting vehicles that are advancing into the curved section in the opposing lane. The system is expected to have the effect of preventing or reducing car accidents and related damage.

Project VIDAS aims to investigate the feasibility of VLC systems with minor complexity, using white and colored LEDs commonly used in outdoor traffic lighting scenarios (namely, vehicle and traffic lights). To achieve this target, several research issues are being addressed: (i) characterization of the outdoor optical communication channel through field measurements, including signal (LEDs) and noise sources; (ii) development of theoretical models of the outdoor visiblelight communication channel (emitter, propagation, receiver and noise sources); (iii) investigation of better positioned modulation schemes adjusted for outdoor VLC systems; and (iv) implementation of an experimental prototype, including the best and cheaper solutions identified.

\section{Concluding Remarks}

Visible Light Communication (VLC) using LEDs can become a viable option for last mile access and ubiquitous availability. Visible Light Communication (VLC) present fascinating challenges for using appropriate techniques to construct cheap processing units and high brightness LEDs. Where LEDs lighting technology is being considered as the next generation lighting devices, VLC using LEDs would be promising technology for ubiquitous communication. The technology promises a great mix of importance, from high energy saving using Solid State Lighting technology and high rate data transmission in indoor applications to traffic safety in outdoor environment. We presented a tutorial of current state-of-art for VLC, investigation and development on VLC WUSB. As explained throughout this article, there remain many research problems. Among them, the most important are in outdoor environment, for overcoming background noise and challenges in cheaper high brightness LEDs. In spite of these open research problems, we believe that the VLC system will be one of the most promising technologies for next-generation optical wireless communication.

Our present work, thorough investigation and prototype development of VLC for traffic safety application under Visible light communication for advanced Driver Assistance System project is ongoing and in the future, car-to-car on road communication and long range parallel communication using VLC will continue. 
Kumar N, et al.: Visible Light Communication Systems

\section{References}

1. M. S. Shur and A. Zukauskas, "Solid-State Lighting: Toward Superior Illumination", Proceedings of the IEEE, vol. 93, October 2005.

2. M. R. Krames, "Introduction to the Issue on High-efficiency LEDs", IEEE Journal on selected topics in Quantum Electronics vol. 8, no. 2, March/April 2002.

3. F. R. Gfeller and U. Bapst, "Wireless In-House Data Communication via Diffuse Infrared Radiation”, Proceedings of the IEEE, vol. 67, Nov. 1979.

4. J. R. Barry, J. M. Kahn, E. A. Lee and D. G. Messerschmitt, "High-Speed Nondirective Optical Communication for Wireless Networks", IEEE Network Magazine, vol. 5, Nov. 1991.

5. J. M. Khan and J. R. Barry, "Wireless Infrared Communications", Proceedings of the IEEE, vol. 85, Feb. 1997.

6. R. L. Aguiar, A. Tavares, J. L. Cura, E. de Vasconcelos, L. N. Alves, R. Valadas, et al., "Considerations on the design of transceivers for wireless optical LANs", IEE Electronics and Communications, Colloquium on Optical Wireless Communications, London, UK, June 1999.

7. M. Akanegawa, Y. Tanaka and M. Nakagawa, "Basic Study on Traffic Information Systems Using LED Traffic Lights", IEEE Transactions on Intelligent Transportation Systems, vol. 2, Dec. 2001.

8. G. Pang, K.-L. Ho, T. Kwan and E. Yang, "Visible Light Communication for Audio Systems", IEEE Transaction on Consumer Electronics, vol. 45, Nov. 1999.

9. G. Pang, T. Kwan, H. Liu and C.-H. Chan, "LED Wireless: A novel use of LEDs to transmit audio and digital signals", IEEE Industry Applications Magazine, vol. 8, Jan. 2002.

10. T. Komine and M. Nakagawa, "Integrated Systems of White LED Visible-Light Communications and Power-Line Communications", IEEE Transactions on Consumer Electronics, vol. 49, Feb. 2003.

11. L. N. Alves and R. L. Aguiar, "Design Techniques for High Performance Optical Wireless Front-Ends", Proceedings of the Conference on Telecommunications - ConfTele 2003, Aveiro, Portugal, April 2003.

12. Toshiko Komine, "Fundamental Analysis for VLC System using LED Lights", IEEE Transactions on Consumer Electronics, vol. 50, no. 1, Feb. 2004.

13. Yuichi T. and M. Nakagawa, "Indoor Visible Light Data Transmission System Utilizing White LEDs Lights”, IEICE Trans, Commun. vol. \#86-B, no. 8, Aug. 2003.

14. H. Sugiyama, S. Haruyama, and M. Nakagawa, "Brightness Control Methods for Illumination and Visible-Light Communication Systems", Proceedings of the Third International Conference on Wireless and Mobile Communications (ICWMC'07).

15. Visible-Light Communication Consortium, http://www.vlcc.net.
16. Y. Hayashi, "Application of Traffic Signals for Wireless Visible Light Communication System," Technical Report of IEICE, ITS2001147, March 2002.

17. S. Iwasaki, M. Wada, T. Endo, T. Fujii and Masayuki, "Tanimoto Basic Experiments on Parallel Wireless Optical Communication for ITS," in Proc. IEEE Intelligent Vehicles Symposium Istanbul, Turkey, June 13-15, 2007.

18. S. Miyauchi, T. Komine, T. Ushiro, S. Yoshimura, S. Haruyama, and M. Nakagawa, "Parallel optical wireless communication using high speed cmos image sensor," Technical Report of IEICE, vol. CS2004-18, May 2004, pp. 7-12.

19. N. Narendran and Y. Gu, "Life of LED-Based White Light Sources", IEEE/OSA Journal of Display Technology, vol. 1, no. 1, Sept. 2005.

20. M. S. Shur and A. R. Zukauskas, "Solid-State Lighting: Toward Superior Illumination", Proceedings of the IEEE, vol. 93, no. 10, Oct. 2005.

21. D. A. Steigerwald, J. C. Bhat, D. Collins, R. M. Fletcher, M. O. Holcomb, M. J. Ludowise, Member, IEEE, Paul S. Martin, and Serge L. Rudaz, "Illumination With Solid State Lighting Technology," IEEE Journal on selected topics in Quantum Electronics, vol. 8, no. 2, March/April 2002.

\section{Appendix -A}

Table 1: The frequency and Switching response of LEDs

\begin{tabular}{lcccc}
\hline Manufacturer & Colour & LED & $\begin{array}{c}\text { Freq. } \\
(\mathrm{MHz})\end{array}$ & $\begin{array}{c}\text { Switching } \\
\text { time }(\mathrm{ns})\end{array}$ \\
\hline Nichia & White & NSSPW500BS & 2,57 & 136,20 \\
& & TCLW5100 & 2,40 & 145,94 \\
Vishay & TLMW3100 & 2,40 & 145,94 \\
& \multirow{4}{*}{ Red } & TLMK3100 & 8,30 & 42,11 \\
& TLCR5100 & 7,23 & 48,35 \\
& TLCR5200 & 7,50 & 46,63 \\
& & TLCR5800 & 7,50 & 46,63 \\
& \multirow{4}{*}{ Green } & TLCY5100 & 5,89 & 59,38 \\
& TLCY5200 & 8,04 & 43,52 \\
& TLCY5800 & 5,89 & 59,38 \\
& TLCTG5100 & 6,53 & 53,54 \\
& Blue & TLCTG5800 & 8,04 & 46,63 \\
& TLCB5100 & 11,75 & 29,76 \\
& TLCB5200 & 12,16 & 28,75 \\
& & TLCB & 12,59 & 27,78
\end{tabular}

\section{AUTHORS}

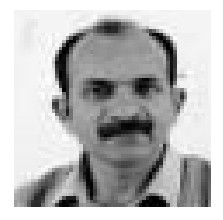

Navin Kumar received degree in Electronics and Telecommunication from IETE, New Delhi in 1995 and ME/M. Tech with specialization in Digital System Engineering in 1999 from Motilal Nehru National Institute of Technology, Allahabad-India. He served in Indian Air Force until 2001 and then faculty member in United College of Engineering and Research, Allahabad. He has worked as expatriate faculty in Addis Ababa University and Defence Engineering College of Ethiopia from 2003 to October 2007. From November 2007 he joined (FCT, Govt of Portugal) funded project on wireless access technology focussing VLC towards his Doctorate degree jointly offered by University of Minho, Aveiro and Porto, Portugal. His research interests include Wireless Optical Communication, VLC, Cooperative and Adhoc Network, Mesh Network. He is the member of IEEE, IET(UK), IETE and IE(India).
E-mail: kumar@av.it.pt

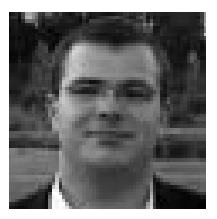

Nuno Rafael M. Lourenço received his B Sc. In electrical engineering from university of Aveiro in 2006 and is a Master student in the department of Electronics, Telecommunication and Information engineering of the university of Aveiro, Portugal. He is preparing his thesis defence in receiver design for VLC.

E-mail: a25417@ua.pt

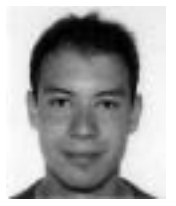

Michal Krzysztof Spiez received his B Sc. In Electrical Engineering from Poland. He has recently completed his Master degree in Electronics and Communication under Erasmus Mundus Master program. He defended his thesis in the university of Aveiro in December 2008 
Kumar N, et al.: Visible Light Communication Systems

in designing Interface for VLC system.

E-mail: mspicz@gmail.com

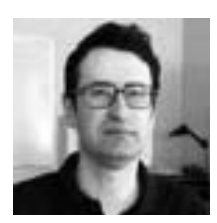

Rui L. Aguiar received the B.Sc. and M.Sc. degrees

in Electronic and Telecommunication engineering from University of Aveiro, Portugal in 1990 and 1995 respectively, and the Ph.D. degree in electronic engineering in 2001. He has executed many European projects in Wireless and Mobile Technology and currently front research leader towards $4 \mathrm{G}$ wireless. He has successfully guided many $\mathrm{PhD}$ and $\mathrm{M} \mathrm{Sc}$ students and has numerous international papers and journals on his name. His research interest includes Access Network, Heterogeneous Network, 4G Technology, Next Generation Network, Circuit and Systems etc. He is currently Associate Professor with the department of Electronics and Telecommunication university of Aveiro.

E-mail: ruilaa@ua.pt

Paper No TR 35_08; Copyright @ 2008 by the IETE 